\title{
Uniform Solution to QSAT Using Polarizationless Active Membranes
}

\author{
Artiom Alhazov ${ }^{1,2}$, Mario J. Pérez-Jiménez ${ }^{3}$ \\ 1 Research Group on Mathematical Linguistics \\ Rovira i Virgili University \\ Pl. Imperial Tarraco 1, 43005 Tarragona, Spain \\ artiome.alhazov@estudiants.urv.cat \\ 2 Institute of Mathematics and Computer Science \\ Academy of Sciences of Moldova \\ Str. Academiei 5, Chişinău, MD 2028, Moldova \\ artiom@math.md \\ 3 Research Group on Natural Computing \\ Department of Computer Science and Artificial Intelligence \\ University of Sevilla \\ Avda. Reina Mercedes s/n, 41012 Sevilla, Spain \\ marper@us.es
}

Summary. It is known that the satisfiability problem (SAT) can be solved a semiuniform family of deterministic polarizationless $\mathrm{P}$ systems with active membranes with non-elementary membrane division. We present a double improvement of this result by showing that the satisfiability of a quantified boolean formula (QSAT) can be solved by a uniform family of $\mathrm{P}$ systems of the same kind.

\section{Introduction}

A particularly interesting class of membrane systems ( $\mathrm{P}$ systems) are the systems with active membranes and with three electrical charges (see [8]), where membrane division can be used in order to solve computationally hard problems in polynomial or even linear time, by a space-time trade-off.

The first efficient semi-uniform solution to SAT (satisfiability problem) was given by Gh. Păun in [8], using division for non-elementary membranes. This result was improved by Gh. Păun, Y. Suzuki, H. Tanaka, and T. Yokomori in [9] using only division for elementary membranes (in that paper also a semi-uniform solution to HPP (hamiltonian path problem) using membrane creation is presented).

P. Sosik in [16] provides an efficient semi-uniform solution to QSAT (quantified satisfiability problem), a well known PSPACE-complete problem, in the framework of $\mathrm{P}$ systems with active membranes but using cell division rules for 
non-elementary membranes. A uniform solution for QSAT was presented in [3], while a semi-uniform polarizationless solution for SAT was presented in [1].

Different efficient uniform solutions have been obtained in the framework of recognizer $\mathrm{P}$ systems with active membranes, with polarizations and only using division rules for elementary membranes ([12], [11], [4], [14], [2], [13], and [15]).

Nevertheless, the polynomial complexity class associated with recognizer P systems with active membranes and with polarizations does not seem precise enough to describe classical complexity classes below PSPACE. Therefore, it is challenging to investigate weaker variants of cell-like membrane systems able to characterize classical complexity classes.

In this paper we work with a variant of these membrane systems that does not use polarizations, and where dissolution rules provide a borderline between efficiency and non-efficiency as shown in [5].

The paper is organized as follows. In the next section some preliminary ideas about recognizer membrane systems and polynomial complexity classes are introduced. In Section 3 we present a uniform and polynomial solution of the quantified satisfiability problem by a family of recognizer $\mathrm{P}$ systems with active membranes, without polarization, permitting dissolution rules and division for elementary and non-elementary membranes. Conclusions and some final remarks are given in Section 4.

\section{Preliminaries}

We start by defining what means to solve decision problems by recognizer P systems, in a semi-uniform or uniform way.

In order to solve $\mathbf{N P}$-complete problems in polynomial (even linear) time in the framework of $\mathrm{P}$ systems without input membrane, we need to design one system for each instance of the problem.

Definition 1. Let $X=\left(I_{X}, \theta_{X}\right)$ be a decision problem. We say that $X$ is solvable in polynomial time by a (countable) family of recognizer membrane systems without input $\boldsymbol{\Pi}=(\Pi(u))_{u \in I_{X}}$, and we denote it by $X \in \mathbf{P} \mathbf{M} \mathbf{C}_{\mathcal{R}}^{*}$, if the following is true:

- The family $\Pi$ is polynomially uniform by Turing machines; that is, there exists a deterministic Turing machine working in polynomial time which constructs the system $\Pi(u)$ from the instance $u \in I_{X}$.

- The family $\Pi$ is polynomially bounded; that is, there exists a polynomial function $p(n)$ such that for each $u \in I_{X}$, all computations of $\Pi(u)$ halt in, at most, $p(|u|)$ steps.

- The family $\boldsymbol{\Pi}$ is sound with regard to $X$; that is, for each instance of the problem $u \in I_{X}$ such that there exists an accepting computation of $\Pi(u)$, we have $\theta_{X}(u)=1$.

- The family $\Pi$ is complete with regard to $X$; that is, for each instance of the problem $u \in I_{X}$ such that $\theta_{X}(u)=1$ every computation of $\Pi(u)$ is an accepting computation. 
We say that the family $\boldsymbol{\Pi}$ is a semi-uniform solution of the problem $X$.

As a direct consequence of working with recognizer membrane systems (all of its computations halt) is that the complexity classes $\mathbf{P} \mathbf{M} \mathbf{C}_{\mathcal{R}}^{*}$ are closed under complement. Moreover, these complexity classes are closed under polynomial time reduction, in the classical sense (see [10]).

Now, we deal with recognizer membrane systems with input membrane solving decision problems in a uniform way in the following sense: all instances of a decision problem with the same size (according to a previously fixed polynomial time computable criterion) are processed by the same system, on which an appropriate input, representing the specific instance, is supplied.

Definition 2. $A$ P system with input membrane is a tuple $\left(\Pi, \Sigma, i_{\Pi}\right)$, where: (a) $\Pi$ is a $P$ system with working alphabet $\Gamma$, with $p$ membranes labeled with $1, \ldots, p$, and initial multisets $\mathcal{M}_{1}, \ldots, \mathcal{M}_{p}$ associated with them; (b) $\Sigma$ is an (input) alphabet strictly contained in $\Gamma$ and the initial multisets are over $\Gamma-\Sigma ;(c) i_{\Pi}$ is the label of a distinguished (input) membrane.

If $m$ is a multiset over the input alphabet $\Sigma$, then the initial configuration of the $P$ system $\left(\Pi, \Sigma, i_{\Pi}\right)$ with input $m$ is $\left(\mu, \mathcal{M}_{1}, \ldots, \mathcal{M}_{i_{\Pi}} \cup m, \ldots, \mathcal{M}_{p}\right)$.

Next, we define the concept of polynomial solvability of decision problems by families of recognizer $\mathrm{P}$ systems constructed in polynomial time by Turing machines.

Definition 3. Let $X=\left(I_{X}, \theta_{X}\right)$ be a decision problem. We say that $X$ is solvable in polynomial time by a family of recognizer membrane systems with input membrane $\boldsymbol{\Pi}=(\Pi(n))_{n \in \mathbf{N}}$, and we denote it by $X \in \mathbf{P M C}_{\mathcal{R}}$, if the following is true:

- The family $\boldsymbol{\Pi}$ is polynomially uniform by Turing machines; that is, there exists a deterministic Turing machine that constructs in polynomial time the system $\Pi(n)$ from $n \in \mathbf{N}$.

- There exists a pair (cod,s) of polynomial-time computable functions whose domain is $L$, such that for each $u \in L, s(u)$ is a natural number and $\operatorname{cod}(u)$ is an input multiset of the system $\Pi(s(u))$, verifying the following:

- The family $\Pi$ is polynomially bounded with regard to $(X, \operatorname{cod}, s)$; that is, there exists a polynomial function $p(n)$ such that for each $u \in I_{X}$ every computation of the system $\Pi(s(u))$ with input $\operatorname{cod}(u)$ is halting and, moreover, it performs at most $p(|u|)$ steps.

- The family $\Pi$ is sound with regard to $(X, c o d, s)$; that is, for each instance of the problem $u \in I_{X}$ such that there exists an accepting computation of $\Pi(s(u))$ with input $\operatorname{cod}(u)$, we have $\theta_{X}(u)=1$.

- The family $\Pi$ is complete with regard to $(X, c o d, s)$; that is, for each instance of the problem $u \in I_{X}$ such that $\theta_{X}(u)=1$, every computation of $\Pi(s(u))$ with input $\operatorname{cod}(u)$ is an accepting one. 
We say that the family $\Pi$ is a uniform solution to the problem $X$.

The complexity classes $\mathbf{P} \mathbf{M C} \mathbf{C}_{\mathcal{R}}$ are closed under complement and under polynomial time reduction, in the classical sense (see [10]).

Let us note that if $\boldsymbol{\Pi}$ is a family of recognizer $\mathrm{P}$ systems solving a decision problem $X$ in polynomial time and in a uniform way, then it provides a polynomial time solution of $X$ in a semi-uniform way. That is, we have $\mathbf{P} \mathbf{M C} \mathbf{C}_{\mathcal{R}} \subseteq \mathbf{P} \mathbf{M} \mathbf{C}_{\mathcal{R}}^{*}$.

\subsection{P systems with polarizationless active membranes}

Definition 4. A P system with active membranes and without polarizations is a $P$ system with $\Gamma$ as working alphabet, with $H$ as the finite set of labels for membranes, and where the rules are of the following forms:

$\left(a_{0}\right)[a \rightarrow u]_{h}$ for $h \in H, a \in \Gamma, u \in \Gamma^{*}$. These are object evolution rules. An object $a \in \Gamma$ belonging to a membrane labeled by $h$ evolves to a string $u \in \Gamma^{*}$.

$\left(b_{0}\right) a[]_{h} \rightarrow[b]_{h}$ for $h \in H, a, b \in \Gamma$. These are send-in communication rules. An object from the region immediately outside a membrane labeled by $h$ is introduced in this membrane, possibly transformed into another object.

$\left(c_{0}\right)[a]_{h} \rightarrow b[]_{h}$ for $h \in H, a, b \in \Gamma$. These are send-out communication rules. An object is sent out from membrane labeled by $h$ to the region immediately outside, possibly transformed into another object.

$\left(d_{0}\right)[a]_{h} \rightarrow b$ for $h \in H, a, b \in \Gamma$. These are dissolution rules. A membrane labeled by $h$ is dissolved in reaction with an object. The skin is never dissolved.

$\left(e_{0}\right)[a]_{h} \rightarrow[b]_{h}[c]_{h}$ for $h \in H, a, b, c \in \Gamma$. These are division rules for elementary membranes. An elementary membrane can be divided into two membranes with the same label, possibly transforming some objects.

$\left(f_{0}\right)\left[[]_{h_{1}}[]_{h_{2}}\right]_{h_{0}} \rightarrow\left[[]_{h_{1}}\right]_{h_{0}}\left[[]_{h_{2}}\right]_{h_{0}}$, where $h_{0}, h_{1}, h_{2}$ are labels. These are division rules for non-elementary membranes. If the membrane with label $h_{0}$ contains other membranes than those with labels $h_{1}, h_{2}$, these membranes and their contents are duplicated and placed in both new copies of the membrane $h_{0}$; all membranes and objects placed inside membranes $h_{1}, h_{2}$, as well as the objects from membrane $h_{0}$ placed outside membranes $h_{1}$ and $h_{2}$, are reproduced in the new copies of membrane $h_{0}$.

These rules are applied according to the following principles:

- All the rules are applied in parallel and in a maximal manner. In one step, one object of a membrane can be used by only one rule (chosen in a nondeterministic way), but any object which can evolve by one rule of any form, must evolve.

- If at the same time a membrane labeled with $h$ is divided by a rule of type $\left(e_{0}\right)$ or $\left(f_{0}\right)$ and there are objects in this membrane which evolve by means of rules of type $\left(a_{0}\right)$, then we suppose that first the evolution rules of type $\left(a_{0}\right)$ are used, and then the division is produced. Of course, this process takes only one step. 
- The rules associated with membranes labeled with $h$ are used for all copies of this membrane. At one step, a membrane can be the subject of only one rule of types $\left(b_{0}\right)-\left(f_{0}\right)$.

We denote by $\mathcal{A M}^{0}(\alpha, \beta)$, where $\alpha \in\{-d,+d\}$ and $\beta \in\{-n e,+n e\}$, the class of all recognizer $\mathrm{P}$ systems with polarizationless active membranes such that:

- if $\alpha=+d$ then dissolution rules are permitted;

- if $\alpha=-d$ then dissolution rules are forbidden;

- if $\beta=+n e$ then division rules for elementary and non-elementary membranes are permitted;

- if $\beta=-n e$ then only division rules for elementary membranes are permitted.

Proposition 1. For each $\alpha \in\{-d,+d\}$ and $\beta \in\{-n e,+n e\}$ we have:

(1) $\mathbf{P M C}_{\mathcal{A M}^{0}(\alpha, \beta)} \subseteq \mathbf{P M C}_{\mathcal{A M}^{0}(\alpha, \beta)}^{*}$.

(2) $\mathbf{P M C}_{\mathcal{A M}^{0}(\alpha,-n e)} \subseteq \mathbf{P M C}_{\mathcal{A M}}{ }^{0}(\alpha,+n e)$.

(3) $\mathbf{P M C}_{\mathcal{A} \mathcal{M}^{0}(\alpha,-n e)}^{*} \subseteq \mathbf{P M C}_{\mathcal{A M}}^{*}(\alpha,+n e)$.

(4) $\mathbf{P M C}_{\mathcal{A M}^{0}(-d, \beta)} \subseteq \mathbf{P M C}_{\mathcal{A M}^{0}(+d, \beta)}$.

(5) $\mathbf{P M C}_{\mathcal{A M}^{0}(-d, \beta)}^{*} \subseteq \mathbf{P M C}_{\mathcal{A M}}^{*}(+d, \beta)$.

A conjecture known in the membrane computing area under the name of the $\mathrm{P}-$ conjecture (proposed by Gh. Păun in 2005) affirms that $\mathbf{P}=\mathbf{P M C}_{\mathcal{A M}^{0}(+d,-n e)}$.

It has been obtained some partial answers of that conjecture ([5]). Specifically, in the framework of recognizer P systems with membrane division but without using polarizations it was shown a surprising role of the dissolution rules, as it makes the difference between efficiency and non-efficiency for $\mathrm{P}$ systems with membrane division and without polarization.

Theorem 1. We have the following:

(1) $\mathbf{P}=\mathbf{P M C}_{\mathcal{A M}^{0}(-d, \beta)}=\mathbf{P M C}_{\mathcal{A M}^{0}(-d, \beta)}^{*}$, for each $\beta \in\{-n e,+n e\}$.

(2) $\mathbf{N P} \subseteq \mathbf{P M C}_{\mathcal{A M}^{0}(+d,+n e)}^{*}$.

\section{A Uniform Solution to QSAT}

In this section we extend the result (2) from Theorem 1, providing an uniform and linear time solution to QSAT (quantified satisfiability) problem, through a family of recognizer $\mathrm{P}$ systems using polarizationless active membranes, dissolution rules, and division for elementary and non-elementary membranes.

Given a boolean formula $\varphi\left(x_{1}, \ldots, x_{n}\right)$ in the conjunctive normal form, with boolean variables $x_{1}, \ldots, x_{n}$, the sentence $\varphi^{*}=\exists x_{1} \forall x_{2} \ldots Q_{n} x_{n} \varphi\left(x_{1}, \ldots, x_{n}\right)$ (where $Q_{n}$ is $\exists$ if $n$ is odd, and $Q_{n}$ is $\forall$ otherwise) is said to be the (existential) fully quantified formula associated with $\varphi\left(x_{1}, \ldots, x_{n}\right)$. Recall that a sentence is a boolean formula in which every variable is in scope of a quantifier. 
We say that $\varphi^{*}$ is satisfiable if there exists a truth assignment, $\sigma$, over $\{i \mid$ $1 \leq i \leq n \wedge i$ odd $\}$ such that each extension, $\sigma^{*}$, of $\sigma$ over $\{1, \ldots, n\}$ verifies $\sigma^{*}\left(\varphi\left(x_{1}, \ldots, x_{n}\right)\right)=1$.

The QSAT problem is the following one: Given the (existential) fully quantified formula $\varphi^{*}$ associated with a boolean formula $\varphi\left(x_{1}, \ldots, x_{n}\right)$ in conjunctive normal form, determine whether or not $\varphi^{*}$ is satisfiable.

It is well known that QSAT is a PSPACE-complete problem [7].

\section{Theorem 2. QSAT belongs to the class $\mathbf{P} \mathbf{M C}_{\mathcal{A M}}(+d,+n e)$.}

Proof. The solution proposed follows a brute force approach, in the framework of recognizer $\mathrm{P}$ systems with polarizationless active membranes where dissolution rules, and division for elementary and non-elementary membranes are permitted. The solution consists in the following stages:

- Generation Stage: using membrane division for elementary and non-elementary membranes, all truth assignments for the variables associated with the boolean formula are produced.

- Assignments stage: in a special membrane we encode the clauses that are satisfied for each truth assignments.

- Checking Stage: we determine what truth assignments make true the boolean formula.

- Quantifier Stage: the universal and existential gates of the fully quantified formula are simulated and its satisfiability is encoded by a special object in a suitable membrane.

- Output Stage: The systems sends out to the environment the right answer according to the result of the previous stage.

Let us consider a propositional formula in the conjunctive normal form:

$$
\begin{aligned}
\varphi & =C_{1} \vee \cdots \vee C_{m}, \\
\operatorname{Var}(\varphi) & =\left\{x_{1}, \ldots, x_{n}\right\} \\
C_{i} & =y_{i, 1} \wedge \cdots \wedge y_{i, l_{i}}, 1 \leq i \leq m, \text { where } \\
y_{i, k} & \in\left\{x_{j}, \neg x_{j} \mid 1 \leq j \leq n\right\}, 1 \leq i \leq m, 1 \leq k \leq l_{i} .
\end{aligned}
$$

We consider a normal form for QSAT: the number of variables is even $\left(n=2 n^{\prime}\right)$ and the quantified formula is

$$
\varphi^{*}=\exists x_{1} \forall x_{2} \ldots \exists x_{n-1} \forall x_{n} \varphi\left(x_{1}, \ldots, x_{n}\right) .
$$

Let us consider the pair function from $\mathbf{N}^{2}$ onto $\mathbf{N}$ defined by

$$
\langle n, m\rangle=((n+m)(n+m+1) / 2)+n .
$$

This function is polynomial time computable and bijective. 
Depending on numbers $m$ (of clauses) and $n$ (of variables), we will consider a system $\left(\Pi(\langle n, m\rangle), \Sigma(\langle n, m\rangle), i_{0}\right)$, where $i_{0}=0$ is the input region and $\Sigma(\langle n, m\rangle)=\left\{v_{i, j}, v_{i, j}^{\prime} \mid 1 \leq i \leq m, 1 \leq j \leq n\right\}$ is the input alphabet.

The problem instance will be encoded in the rules of the P system by multisets $X$ and $X^{\prime}$ of symbols from $\Sigma$, corresponding to the clause-variable pairs such that the clause is satisfied by true and false assignment of the variable:

$$
\begin{aligned}
X & =\left\{\left(v_{i, j}, 1\right) \mid x_{j} \in\left\{y_{i, k} \mid 1 \leq k \leq l_{i}\right\}, 1 \leq i \leq m, 1 \leq j \leq n\right\}, \\
X^{\prime} & =\left\{\left(v_{i, j}^{\prime}, 1\right) \mid \neg x_{j} \in\left\{y_{i, k} \mid 1 \leq k \leq l_{i}\right\}, 1 \leq i \leq m, 1 \leq j \leq n\right\} .
\end{aligned}
$$

We now construct the P system

$$
\begin{aligned}
\Pi(\langle n, m\rangle) & =\left(O, H, \mu, w_{0}, \cdots, w_{m+5 n+3}, R\right), \text { with } \\
O & =\Sigma(\langle n, m\rangle) \cup\left\{u_{i, j}, u_{i, j}^{\prime} \mid 1 \leq i \leq m, 1 \leq j \leq n\right\} \\
& \cup\left\{d_{i} \mid 0 \leq i \leq 2 m+7 n+2\right\} \cup\left\{a_{i}, t_{i}, f_{i} \mid 1 \leq i \leq n\right\} \\
& \cup\left\{c_{i} \mid 1 \leq i \leq m\right\} \cup\left\{t, f, z, z^{\prime}, T, T^{\prime}, \text { yes, no }\right\}, \\
\mu & =\left[\left[\cdots\left[[]_{0}\right]_{1} \cdots\right]_{m+5 n+2}\right]_{m+5 n+3}, \\
w_{0} & =w_{m+5 n+1}=d_{0}, \\
w_{m+2 n+3 i} & =d_{m+5 n-2}, 1 \leq i \leq n, \\
w_{i} & =\lambda, i \notin\{0, m+5 n+1\} \cup\{m+2 n+3 i \mid 1 \leq i \leq n\}, \\
H & =\{0, \cdots, m+5 n+3\},
\end{aligned}
$$

and the following rules (we also explain their use):

\section{Generation stage}

$$
\begin{aligned}
& \mathrm{G} 1\left[d_{3 i} \rightarrow a_{i+1} d_{3 i+1}\right]_{0},\left[d_{3 i+1} \rightarrow d_{3 i+2}\right]_{0},\left[d_{3 i+2} \rightarrow d_{3 i+3}\right]_{0}, 0 \leq i<n . \\
& \quad\left[d_{3 n+i} \rightarrow d_{3 n+i+1}\right]_{0}, 0 \leq i<m+2 n .
\end{aligned}
$$

We count to $m+5 n$, which is the time needed for producing all $2^{n}$ truth assignments for the $n$ variables, as well as membrane sub-structures which will examine the truth value of formula $\varphi$ for each of these truth assignments; this counting is done in the central membrane; moreover during steps $3 i, 0 \leq i \leq n$, symbols $a_{1}, \cdots, a_{n}$ are subsequently produced.

$$
\mathrm{G} 2\left[a_{i}\right]_{0} \rightarrow\left[t_{i}\right]_{0}\left[f_{i}\right]_{0}, 1 \leq i \leq n .
$$

In membrane 0 , we subsequently choose each variable $x_{i}, 1 \leq i \leq n$, and both values true and false are associated with it, in form of objects $t_{i}$ and $f_{i}$, which are separated in two membranes with label 0 . The division of membrane 0 is triggered by the objects $a_{i}$, which are introduced by the first rule from group G1 in odd steps; this is important in interleaving the use of these rules (hence the division of membrane 0) with the use of the rules of group G4, for dividing membranes placed above membrane 0 . 


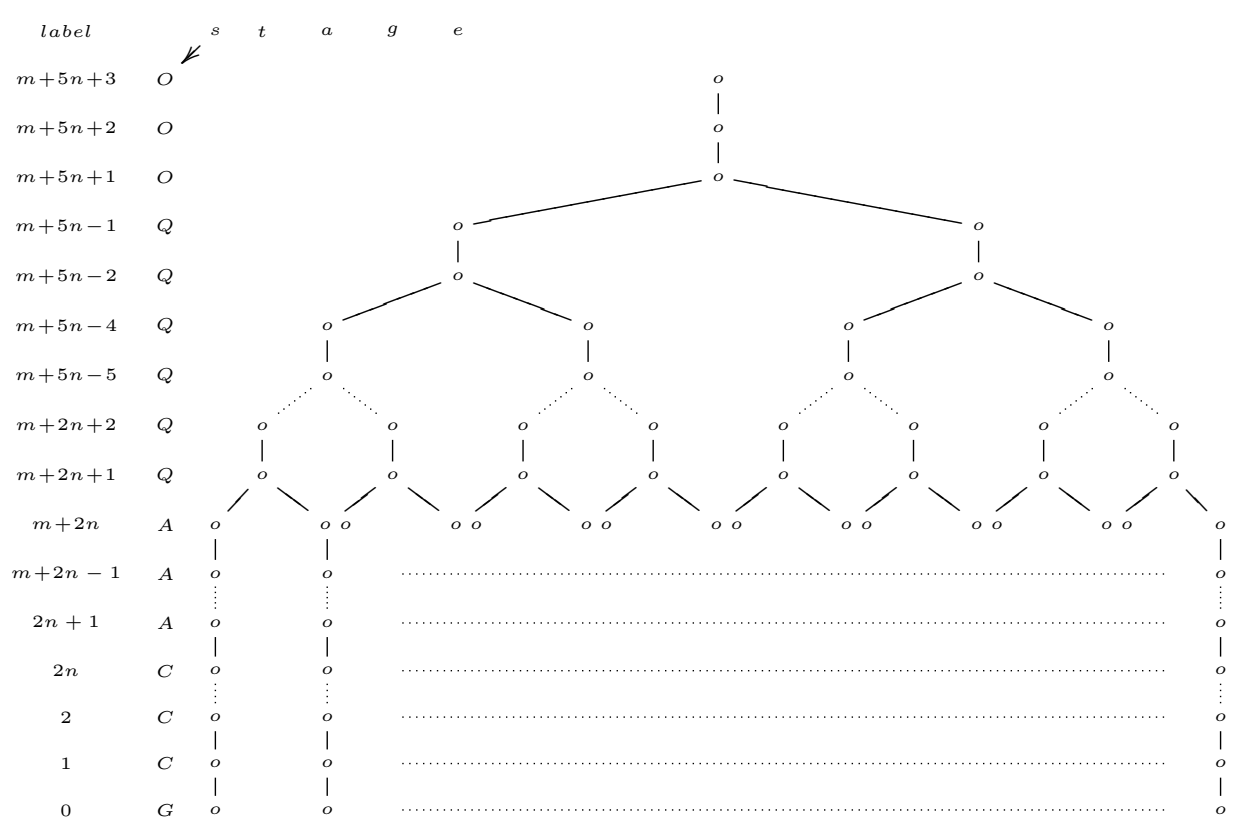

Fig. 1. The membrane structure of the system $\Pi$ after $m+5 n$ steps.

$$
\begin{aligned}
& \mathrm{G} 3\left[d_{j} \rightarrow d_{j-1}\right]_{m+2 n+3 i}, 1 \leq j \leq m+5 n-2,1 \leq i<j, \\
& \quad\left[d_{0}\right]_{m+2 n+3 i} \rightarrow z^{\prime}, 1 \leq i \leq n .
\end{aligned}
$$

After $m+5 n-2$ steps, dissolution rules are applied to membranes $m+2 n+3 i$.

$$
\mathrm{G} 4\left[[]_{i}[]_{i}\right]_{i+1} \rightarrow\left[[]_{i}\right]_{i+1}\left[[]_{i}\right]_{i+1}, 0 \leq i<m+5 n .
$$

These are division rules for membranes with label $0,1, \cdots, m+5 n$, to be used for the central membrane 0 in steps which follow the use of the first rule of type G1. The division of a membrane with label 1 is then propagated from lower levels to upper levels of the membrane structure and the membranes are continuously divided. The membrane division stops at the level where a membrane $m+2 n+3 i$ has been already dissolved by a rule from group G3. This results in the structure as shown in Figure 1 after $m+5 n$ steps.

$$
\text { G5 }\left[d_{m+5 n}\right]_{0} \rightarrow T \text {. }
$$

After $m+5 n$ steps, each copy of membrane with label 0 is dissolved and the contents is released into the surrounding membrane, which is labeled with 1 .

\section{Assignments stage}

$$
\begin{gathered}
\mathrm{A} 1\left[t_{i} \rightarrow t^{\prime}\right]_{2 i-1}, \\
\quad\left[t^{\prime}\right]_{2 i-1} \rightarrow z, \\
\quad\left[f_{i}\right]_{2 i-1} \rightarrow f^{\prime},
\end{gathered}
$$




$$
\begin{aligned}
& {\left[f^{\prime} \rightarrow z\right]_{2 i},} \\
& {[z]_{2 i} \rightarrow z^{\prime}, 1 \leq i \leq n .}
\end{aligned}
$$

Depending on the variable assignments, we need to determine what clauses are satisfied. For a variable $x_{i}$, this is done in membranes $2 i-1$ and $2 i$. The objects encoding the problem propagate through the membrane structure: object $t_{i}$ dissolves membrane $2 i-1$ after one step, and then it dissolves membrane $2 i$ immediately, while object $f_{i}$ dissolves membrane $2 i-1$ immediately, and then it dissolves membrane $2 i$ after one step.

$$
\begin{array}{r}
\mathrm{A} 2\left[v_{i, j} \rightarrow u_{i, j}\right]_{2 i-1}, \\
{\left[v_{i, j}^{\prime} \rightarrow u_{i, j}^{\prime}\right]_{2 i-1}}
\end{array}
$$

Once in membrane $2 i-1$, objects $v_{i, j}$ and $v_{i, j}^{\prime}$ wait for one step.

$$
\begin{aligned}
\mathrm{A} 3 & {\left[u_{i, j}^{\prime} \rightarrow \lambda\right]_{2 i-1}, } \\
& {\left[u_{i, j} \rightarrow c_{i}\right]_{2 i-1}, } \\
& {\left[u_{i, j} \rightarrow \lambda\right]_{2 i}, } \\
& {\left[u_{i, j}^{\prime} \rightarrow c_{i}\right]_{2 i}, 1 \leq i \leq n . }
\end{aligned}
$$

If membrane $2 i-1$ is not dissolved in the meantime, then the objects encoding the instance of SAT evolve according to the value true of $x_{i}$, otherwise, they evolve according to the value false of $x_{i}$.

At the end of this routine (it takes $3 n$ steps), a membrane with label $2 n+1$ which contains all the symbols $c_{1}, \cdots, c_{m}$ corresponds to the truth assignment satisfying all clauses, hence it satisfies formula $\varphi$, and vice-versa.

\section{Checking stage}

$$
\mathrm{C} 1\left[c_{i}\right]_{2 n+i} \rightarrow c_{i}, 1 \leq i \leq m .
$$

A membrane with label $2 n+i, 1 \leq i \leq m$, is dissolved if and only if $c_{j}$ appears in it (i.e., clause $C_{j}$ is satisfied by the current truth assignment); if this is the case, the truth assignment associated with the membrane is released in the surrounding membrane. Otherwise, the truth assignment remains blocked in membrane $2 n+i$ and never used at the next steps by the membranes placed above.

$$
\mathrm{C} 2[T]_{m+2 n+1} \rightarrow T \text {. }
$$

The fact that the object $T$ appears in the membrane with the label $m+2 n+1$ means that there is a truth assignment which satisfies the formula $\varphi$. In this case, the membrane with label $m+2 n+1$ is dissolved and the contents are released into the membrane with label $m+2 n+2$. Otherwise, the formula is not satisfiable, and the membrane with label $m+2 n+1$ will not dissolve.

\section{Quantifier stage}

$$
\begin{aligned}
& \mathrm{Q} 1[T]_{m+2 n+6 i+1} \rightarrow T^{\prime}, \\
& \quad[T]_{m+2 n+6 i+2} \rightarrow T, \\
& \quad\left[T^{\prime} \rightarrow \lambda\right]_{m+2 n+6 i+2}, 1 \leq 2 i \leq m .
\end{aligned}
$$


The universal gate of the formula is simulated by dissolution of two membranes: this happens if and only if two copies of $T$ are present. One copy dissolves membrane $m+2 n+6 i+1$ and is erased while the other copy dissolves membrane $m+2 n+6 i+2$ and sends one copy of $T$ outside; otherwise the computation in this gate stops without sending any object out. Recall that membrane $m+2 n+6 i+3$ has been erased by rule from group G3.

$$
\begin{aligned}
\mathrm{Q} 2 & {[T]_{m+2 n+6 i+4} \rightarrow T^{\prime}, } \\
& {\left[T^{\prime}\right]_{m+2 n+6 i+5} \rightarrow T, } \\
& {[T \rightarrow \lambda]_{m+2 n+6 i+5}, 1 \leq 2 i \leq m . }
\end{aligned}
$$

The existential gate of the formula is simulated by the dissolution of two membranes: this happens if and only at least one copy of $T$ is present. One copy dissolves membrane $m+2 n+6 i+4$ and then it also dissolves membrane $m+2 n+6 i+2$ (thus sending one copy of $T$ outside), while the other copy (if exists) is erased; if no copy of $T$ is present, no rule is applied, so the gate sends nothing outside. Recall that membrane $m+2 n+6 i+6$ has been erased by rule from group G3.

$$
\text { Q3 }\left[d_{i} \rightarrow d_{i+1}\right]_{m+5 n+1}, 0 \leq i \leq 2 m+8 n+1 .
$$

At the same time as the membrane with label $m+5 n+1$ is dissolved (at step $2 m+8 n+1)$, the object $d_{2 m+8 n+1}$ evolves to $d_{2 m+8 n+2}$, and then released to the membrane with label $m+5 n+2$.

\section{Output stage}

$$
\begin{aligned}
& \text { O1 }\left[d_{2 m+8 n+2}\right]_{m+5 n+2} \rightarrow \text { yes. } \\
& \mathrm{O} 2[a]_{m+8 n+3} \rightarrow[]_{m+5 n+3} a, a \in\{\text { yes, no }\} .
\end{aligned}
$$

In the next two steps, the object yes is produced, and then send out to the environment.

$$
\begin{aligned}
& \text { O3 }\left[d_{2 m+8 n+2}\right]_{m+5 n+1} \rightarrow \text { no. } \\
& \text { O4 }[\mathrm{no}]_{m+5 n+2} \rightarrow \text { no. }
\end{aligned}
$$

If the formula is not satisfiable, then the object $d_{2 m+8 n+1}$ remains in the membrane with label $m+5 n+1$, which produces the object $n o$, ejecting it into the membrane with label $m+5 n+2$, then into the membrane with label $m+5 n+3$, finally into the environment.

Therefore, in step $2 m+8 n+3$ the system halts and sends into the environment one of the objects yes, no, indicating whether or not the formula $\varphi^{*}$ is satisfiable.

It is easy to see that the system $\Pi$ can be constructed in a polynomial time starting from numbers $m, n$, and this concludes the proof.

Remark 1. The systems constructed above are deterministic.

Remark 2. It is possible to speed up the system; the present construction is made for an easier explanation: the stages do not overlap in time. 
Remark 3. Notice that the only rules of type $\left(c_{0}\right)$ in the system are rules $\mathrm{O} 2$, that are executed in the last step of the computation. Hence, these rules are not important for deciding whether $\varphi^{*}$ is satisfied; they are only needed to send the answer out of the skin membrane.

From Theorem 2, and having in mind that the complexity class $\mathbf{P M C}_{\mathcal{A M}^{0}(+d,+n e)}$ is closed under polynomial time reductions, we have the following result.

\section{Corollary 1. PSPACE $\subseteq \mathbf{P M C}_{\mathcal{A M}^{0}(+d,+n e)}$.}

\section{Conclusions}

The framework of recognizer $\mathrm{P}$ systems with active membranes and with three electrical charges does not seem precise enough to describe classical complexity classes below PSPACE. In ([5], [6]) weaker variants of these P systems removing polarizations but keeping other usual ingredients associated with active membranes (no cooperation, no priorities, and without changing the labels of membranes) have been considered. It was shown ([5]) that in the above framework, but do not using dissolution rules, it is possible to solve in polynomial time only decision problems which are tractable in the standard sense. Moreover, if we consider membrane dissolution rules then we can solve $\mathbf{N P}$-complete problems in polynomial time, in a semi-uniform way, and using division for elementary and non-elementary membranes ([5]).

In this paper we give a polynomial time and uniform solution to QSAT, a well known PSPACE-complete problem, through a family of recognizer P systems using polarizationless active membranes, dissolution rules and division for elementary and non-elementary membranes. It remain as an open question if the division for non-elementary membranes can be removed.

\section{Acknowledgements}

The first author is supported by the project TIC2003-09319-C03-01 of the Research Group on Mathematical Linguistics, Tarragona. The second author wishes to acknowledge the support of the project TIN2005-09345-C04-01 of the Ministerio de Educación y Ciencia of Spain, cofinanced by FEDER funds.

\section{References}

1. A. Alhazov, L. Pan, Gh. Păun: Trading polarizations for labels in P systems with active membranes. Acta Informaticae, 41, 2-3 (2004), 111-144.

2. A. Alhazov, C. Martín-Vide, L. Pan: Solving graph problems by P systems with restricted elementary active membranes. In Aspects of Molecular Computing (N. Jonoska, Gh. Păun, G. Rozenberg, eds.), LNCS 2950, Springer, 2004, 1-22. 
3. A. Alhazov, C. Martín-Vide, L. Pan: Solving a PSPACE-complete problem by P systems with restricted active membranes. Fundamenta Informaticae, 58, 2 (2003), 67-77.

4. M.A. Gutiérrez-Naranjo, M.J. Pérez-Jiménez, A. Riscos-Núñez: A fast P system for finding a balanced 2-partition. Soft Computing, 9, 9 (2005), 673-678.

5. M.A. Gutiérrez-Naranjo, M.J. Pérez-Jiménez, A. Riscos-Núñez, F.J. RomeroCampero: On the power of dissolution in $\mathrm{P}$ systems with active membranes. In Membrane Computing, 6th International Workshop, WMC 2005 (R. Freund, Gh. Păun, G. Rozenberg, A. Salomaa, eds.), LNCS 3850, Springer, 2006, 224-240.

6. M.A. Gutiérrez-Naranjo, M.J. Pérez-Jiménez, A. Riscos-Núñez, F.J. RomeroCampero: P systems with active membranes, without polarizations and without dissolution: A characterization of P. In Unconventional Computation, 4th International Conference, UC 2005 (C.S. Calude, M.J. Dinneen, Gh. Păun, M.J. Pérez-Jiménez, G. Rozenberg, eds.), LNCS 3699, Springer, 2005, 105-116.

7. C.H. Papadimitriou: Computational Complexity. Addison-Wesley, 1995.

8. Gh. Păun: P systems with active membranes: Attacking NP-complete problems. Journal of Automata, Languages and Combinatorics, 6, 1 (2001), 75-90.

9. Gh. Păun, Y. Suzuki, H. Tanaka, T. Yokomori: On the power of membrane division in P systems. Theoretical Computer Science, 324, 1 (2004), 61-85.

10. M.J. Pérez-Jiménez: An approach to computational complexity in membrane computing. In Membrane Computing (G. Mauri, Gh. Păun, M. J. Pérez-Jiménez, G. Rozenberg, A. Salomaa, eds.), LNCS 3365, Springer, 2005, 85-109.

11. M.J. Pérez-Jiménez, A. Riscos-Núñez: Solving the Subset-Sum problem by active membranes. New Generation Computing, 23, 4 (2005), 367-384.

12. M.J. Pérez-Jiménez, A. Riscos-Núñez: A linear-time solution to the Knapsack problem using $\mathrm{P}$ systems with active membranes. In Membrane Computing (C. MartínVide, Gh. Păun, G. Rozenberg, A. Salomaa, eds.), LNCS 2933, Springer, 2004, 250268.

13. M.J. Pérez-Jiménez, F.J. Romero-Campero: Solving the Bin Packing problem by recognizer $\mathrm{P}$ systems with active membranes. In Proceedings of the Second Brainstorming Week on Membrane Computing (Gh. Păun, A. Riscos-Núñez, A. RomeroJiménez, F. Sancho-Caparrini, eds.), Report RGNC 01/04, University of Seville, 2004, 414-430.

14. M.J. Pérez-Jiménez, A. Romero-Jiménez, F. Sancho-Caparrini: Complexity classes in cellular computing with membranes. Natural Computing, 2, 3 (2003), 265-285.

15. M.J. Pérez-Jiménez, F.J. Romero-Campero: Attacking the Common Algorithmic Problem by recognizer $\mathrm{P}$ systems. In Machines, Computations and Universality (M. Margenstern, ed.), LNCS 3354, Springer, 2005, 304-315.

16. P. Sosik: The computational power of cell division. Natural Computing, 2, 3 (2003), $287-298$. 